\title{
Genotypic Diversity and Characterization of Quinolone Resistant Determinants from Enterobacteriaceae in Yaoundé, Cameroon
}

\author{
Emilia Enjema Lyonga Mbamyah ${ }^{1,2 *}$, Michel Toukam¹, Marie-Claire Okomo Assoumou1,2, \\ Anthony M. Smith ${ }^{3}$, Celine Nkenfou ${ }^{4}$, Hortense Kamga Gonsu, 1,5, Anicette Chafa Betbeui1,5, \\ Martha Tongo Mesembe ${ }^{2}$, Agnes Bedie Eyoh ${ }^{2}$, George Mondinde Ikomey ${ }^{1,2}$, Sinata Koulla-Shiro ${ }^{1}$ \\ ${ }^{1}$ Faculty of Medicine and Biomedical Sciences, University of Yaoundé 1, Yaoundé, Cameroon \\ ${ }^{2}$ Centre for the Study and Control of Communicable Diseases, Faculty of Medicine and Biomedical Sciences, University of \\ Yaoundé 1, Yaoundé, Cameroon \\ ${ }^{3}$ Center for Enteric Diseases, National Institute for Communicable Diseases, Johannesburg, South Africa \\ ${ }^{4}$ Higher Teachers' Training College, University of Yaoundé 1, Yaoundé, Cameroon \\ ${ }^{5}$ University Teaching Hospital, Yaoundé, Cameroon \\ Email: *emilialyo@yahoo.co.uk
}

How to cite this paper: Lyonga Mbamyah, E.E., Toukam, M., Assoumou, M.-C.O. Smith, A.M., Nkenfou, C., Gonsu, H.K., Betbeui, A.C., Mesembe, M.T., Eyoh, A.B. Ikomey, G.M. and Koulla-Shiro, S. (2020) Genotypic Diversity and Characterization of Quinolone Resistant Determinants from Enterobacteriaceae in Yaoundé, Cameroon. Open Journal of Medical Microbiology, 10, 33-45

https://doi.org/10.4236/ojmm.2020.102004

Received: February 5, 2020

Accepted: May 8, 2020

Published: May 11, 2020

Copyright $\odot 2020$ by author(s) and Scientific Research Publishing Inc. This work is licensed under the Creative Commons Attribution International License (CC BY 4.0).

http://creativecommons.org/licenses/by/4.0/

Open Access

\begin{abstract}
Background: Enterobacteriaceae causes many types of infections which are often treated with quinolones and fluoroquinolone $(\mathrm{Q} / \mathrm{FQ})$. The resistance mechanisms to $\mathrm{Q} / \mathrm{FQ}$ are usually associated with mutations in the quinolone resistance determining region which alter the conformation of target amino acid residues within the protein and in the qnr genes. This study aimed at determining the antimicrobial resistant profile of a sample of Enterobacteriaceae from Cameroon and the genetic diversity in quinolone-resistant isolates in view of implementing a better management, treatment, control and prevention of the transmission of these resistant strains. Methods: Identification and antimicrobial susceptibility testing was done using VITEK 2 . The detection of plamid-mediated quinolone resistance (PMQR) genes was carried out using the conventional PCR method. Sequencing was done using the Applied Biosystem 3500 genetic analyser. DNA fingerprint was obtained using Pulsed-Field Gel electrophoresis. Results: Among 440 Enterobacteriaceae, the most prevalent genera were: Escherichia 178/440 (39.5\%); Klebsiella 148/440 (33.6\%); Enterobacter 35/440 (8\%); Pantoea 28/440 (6.4\%); Proteus 14/440 (3.2\%) Salmonella 13/440 (3\%). Ampicillin resistance showed the highest prevalence with $371 / 440(81 \%)$ and Imipenem the lowest resistance $9 / 440$ (2.1\%). The ciprofloxacin resistance rate was 161/440 (36.6\%). The detected plasmid mediated quinolone resistance (PMQR) genes were: qnrA, 2/161 (1.2\%); qnrB, 31/161 (19.3\%); qnrS, 13/161 (8.1\%): Aac (6)Ib-cr, 84/161
\end{abstract}


(52.2\%) and $q e p A, 3 / 161$ (1.9\%). There were several mutations in the parC gene of Klebsiella leading to S80D and S80N substitutions. Two pairs of Klebsiella peumoniae strains were phenotypically and genotypically identical with $100 \%$ similarity in the dendrogramme. Conclusion: This study showed that quinolone resistance was high. The PMQR genes contributing to this resistance were diverse. This high PMQR indicates that there has been an unknown circulation of these genes in our community. To avoid the rapid dissemination of these PMQR genes continuous surveillance of antimicrobial resistance should be carried out not only in humans but also in animals to monitor the evolution of these genes.

\section{Keywords}

Enterobacteriaceae, Quinolone Resistance, Plasmid-Mediated Quinolone Resistance, $q n r$ Genes

\section{Introduction}

The Enterobacteriaceae is a very important family of bacteria with over $80 \%$ of the isolates being of medical importance among the Gram-negative bacilli [1]. Most infections caused by this family are often treated using the Fluoroquinolones (FQ). The use of this class of antimicrobial accounts for up to $11 \%$ of the total prescriptions of antimicrobials in human medicine globally [2] [3].

Early predictions suggested that the emergence of FQ resistance, particularly among the Enterobacteriaceae, was very unlikely. Researchers thought that FQ resistance was unlikely to evolve, mainly because resistant Escherichia coli mutants are readily selected in vitro and because plasmid-mediated quinolone resistance (PMQR) remained unknown even after 30 years of nalidixic acid usage. Subsequent reports noting the emergence of FQ resistance in the Enterobacteriaceae were of great concern, given that these pathogens cause a substantial proportion of serious hospital-acquired infections [4].

Most recently, FQ resistance has emerged in E. coli and other Enterobacteriaceae, contingent on multiple mutations that diminish the affinity of its type II topoisomerase in various ways, reduce permeability, and upregulate efflux [5] [6].

Today, antimicrobial resistance is one of the major problems faced by clinicians in their work. The discovery and rapid dissemination of plasmid-mediated quinolone resistance (PMQR) genes have further highlighted the problem of quinolone and FQ resistance [2]. The emergence of strains showing resistance to several quinolone antimicrobial agents is a public health concern [7].

The resistance mechanisms to quinolones are usually associated with mutations in the quinolone-resistance determining region (QRDR) of gyrA, corresponding to the amino-terminal segment (position 67-106), with increased resistance associated with mutations in a similar region of $\operatorname{parC}$ and acquisition of the qnr genes and other PMQR genes that substitute one target amino acid for 
another within the protein [3] [7] [8].

The detection of such horizontally transferable genes helps understand the close association between resistance to quinolone and resistances to other agents. It explains how the low-level quinolone resistance shown in vitro facilitates the emergence of higher-level resistance in the presence of quinolone at therapeutic levels [9].

In Cameroon, quinolone resistance among Gram-negative bacilli and Enterobacteriaceae was $12 \%$ and $25.7 \%$ among Enterobacteriaceae [10] [11] [12] [13] respectively. However, little data were found on the detection of PMQR genes among Enterobacteriaceae.

This study aimed at determining the genetic diversity in quinolone resistance genes of Enterobacteriaceae in view of implementing better management, control, treatment and prevention of the transmission of these resistant strains in Cameroon. The data generated from this study would provide information on the epidemiology of Enterobacteriaceae species, the antimicrobial resistance profile and report the detection of PMQR genes in Cameroon.

\section{Methods}

\subsection{Bacterial Isolation and Identification}

Four hundred and forty clinical specimens were collected from June 2013 to June 2015 from in- and out-patients at three referral hospitals in Yaounde: The Yaounde University Teaching Hospital, Yaounde General Hospital and the Yaoundé Gynaeco-Obstetric and Pediatric Hospital. The specimens included urine, vaginal and cervical smear, blood, urinary catheter, pus, stool, sputum, bed sore, pleural fluid, seminal fluid and wound. The specimens were cultured in a Gram negative selective media, eosin methylene blue (EMB). Isolated colonies were confirmed to be Gram negative bacilli through Gram staining. The identification of Enterobacteriaceae species was done using the Gram-negative (GN) cards of the VITEK 2 COMPACT (BioMérieux SA, Marcy-Etoile, Lyon, France) by following the manufacturer's procedure carefully.

\subsection{Antimicrobial Susceptibility Testing}

Antimicrobial susceptibility testing (AST) was done using the AST-N255 cards of the VITEK 2 COMPACT (BioMérieux SA, Marcy-Etoile, Lyon, France). Quinolone resistance (QR) in the Enterobacteriaceae was confirmed by determining the minimum inhibitory concentration (MIC) for ciprofloxacin using the E-test (BioMérieux SA, Marcy-Etoile, Lyon, France). The breakpoint values were determined as prescribed by the Clinical Laboratory Standard Institutes (CLSI) 2016 performance guidelines for AST [14]. The control strain, Escherichia coli ATCC 25922 was used as quality control for the VITEK and the E-test.

\subsection{PCR Detection of PMQR Genes}

Extraction of crude genomic DNA from the quinolone-resistant Enterobacteria- 
ceae was carried out using the boiling method as previously described [3]. The detection, prevalence and diversity of PMQR genes were carried out using conventional PCR. Table 1 presents the list of genes and primer sequences used for the PCR amplification of the PMQR genes. The amplification conditions were those previously described [3] [7] on a DNA Engine Tetrad (Bio-Rad, Marnesla-Coquette, France) using a Super-Therm Gold DNA Polymerase (Southern Cross Biotechnology). The resulting PCR amplicons were analysed by electrophoresis on $1.5 \%$ agarose gel containing ethidium bromide and visualized by ultra violet (UV) light.

\subsection{DNA Sequencing and Molecular Subtyping}

The amplicons were purified using the INVITEK kit (STRATEC Molecular $\mathrm{GMbH}$, Berlin, Germany).The resulting product was then sequenced separately using the forward and reverse primers with the Big Dye Terminator Kit (Applied Biosystems, Carlsbad, California, USA). Sequencing products were cleaned up using Dye Ex Spin kit (Qiagen Inc., Valencia, CA, USA). The cleaned product was vacuum dried for one hour at a temperature of $30^{\circ} \mathrm{C}$ under aqueous condition.

Pulsed-Field Gel Electrophoresis (PFGE) was used to investigate the genetic relatedness of plasmid-mediated quinolone resistant strains from different individuals. PFGE was carried out for selected PMQR strains with at least one resistance gene detected. The DNA was digested using the $X b a 1$ enzyme and electrophoresis

Table 1. The PMQR primers used in this study.

\begin{tabular}{|c|c|c|c|}
\hline Gene & Primer name & Primer sequence & Expected size (bp) \\
\hline \multirow[t]{3}{*}{ QnrA } & $q n r A-\mathrm{F}$ & TCAGCAAGAGGATTTCTCA & \multirow{3}{*}{627} \\
\hline & & & \\
\hline & $q n r A-\mathrm{R}$ & GGCAGCACTATTACTCCCA & \\
\hline \multirow[t]{2}{*}{$Q n r B$} & $q n r B-\mathrm{F}$ & GATCGTGAAAGCCAGAAAGG & \multirow{2}{*}{476} \\
\hline & $q n r B-\mathrm{R}$ & ACGATGCCTGGTAGTTGTCC & \\
\hline \multirow[t]{2}{*}{ QnrS } & $q n r S-\mathrm{F}$ & ATGGAAACCTACAATCATAC & \multirow{2}{*}{491} \\
\hline & $q n r S-\mathrm{R}$ & AAAAACACCTCGACTTAAGT & \\
\hline \multirow[t]{2}{*}{$A a c(6) I b-c r$} & $a a c(6) I b-c r-F$ & TTGCGATGCTCTATGAGTGGCTA & \multirow{2}{*}{482} \\
\hline & $a a c(6) I b-c r-\mathrm{R}$ & CTCGAATGCCTGGCGTGTTT & \\
\hline \multirow[t]{2}{*}{ QepA } & $q e p A-\mathrm{F}$ & GCAGGTCCAGCAGCGGGTAG & \multirow{2}{*}{199} \\
\hline & $q e p A-\mathrm{R}$ & CTTCCTGCCCGAGTATCGTG & \\
\hline \multirow[t]{2}{*}{ GyrA } & gyrA-F & CGACCTTGCGAGAGAAAT & \multirow{2}{*}{625} \\
\hline & gyrA-R & GTTCCATCAGCCCTTCAA & \\
\hline \multirow[t]{2}{*}{ parC-E. coli } & $\operatorname{par} C$-E-F & AAACCTGTTCAGCGCCGCATT & \multirow{2}{*}{395} \\
\hline & $\operatorname{par} C-\mathrm{E}-\mathrm{R}$ & GTGGTGCCGTTAAGCAAA & \\
\hline \multirow[t]{2}{*}{ parC-Klebsiella } & $\operatorname{parC}-\mathrm{K}-\mathrm{F} 1$ & CTGAATGCCAGCGCCAAATT & \multirow{2}{*}{389} \\
\hline & $\operatorname{par} C-\mathrm{K}-\mathrm{R} 1$ & TGCGGTGGAATATCGGTCGC & \\
\hline
\end{tabular}

Source: Minh Vien et al., 2009, Stephenson et al., 2010. 
was carried out using the CHEF-DR III electrophoresis system (Bio-Rad Laboratories Inc., USA). The PFGE patterns were captured using the BIORAD gel documentation system (quantity one software). The patterns were further analyzed using the Applied Maths Bionumerics software.

\section{Results}

\subsection{Bacterial Isolation and Identification}

The prevalence of the Enterobacteriaceae by genus was Escherichia, 178/440 (39.5\%); Klebsiella, 148/440 (33.6\%); Enterobacter, 35/440 (8\%); Pantoea 28/440 (6.4\%); Salmonella 13/440 (3\%); Serratia 11/440 (2.5\%); Yersinia and Citrobacter both having $7 / 440(1.6 \%)$ each and Shigella $2 / 440(0.5 \%)$.

\subsection{Antimicrobial Susceptibility Testing}

The resistance pattern of the 440 isolates to the twelve antimicrobials tested is shown on Figure 1.

The isolates were most resistant to ampicillin (AM) with a resistant rate of $371 / 440$ (81\%), followed by cefuroxime axetil (XM-A) with 213/440 (48\%), amoxicillin/clavulanic acid (XL) with 209/440 (47\%), cefuroxime (XM) with 198/440 (45\%), gentamicin (GM) 191/440 (43.3\%), ciprofloxaxin 165/440 (CI) (37.5\%), ceftazidime (TZ) 153/440 34.8\%, cefotaxime (CT) 151/440 (34.3\%), piperacillin/tazobactam (PT) 84/440 (19.1\%), and amikacin 80/440 (18.2\%).

The isolates were most sensitive to imipenem with only $9 / 440(2.1 \%)$ of the isolates being resistant and nitrofurantoin (NI) with 70/440 (15.9\%) resistant isolates.

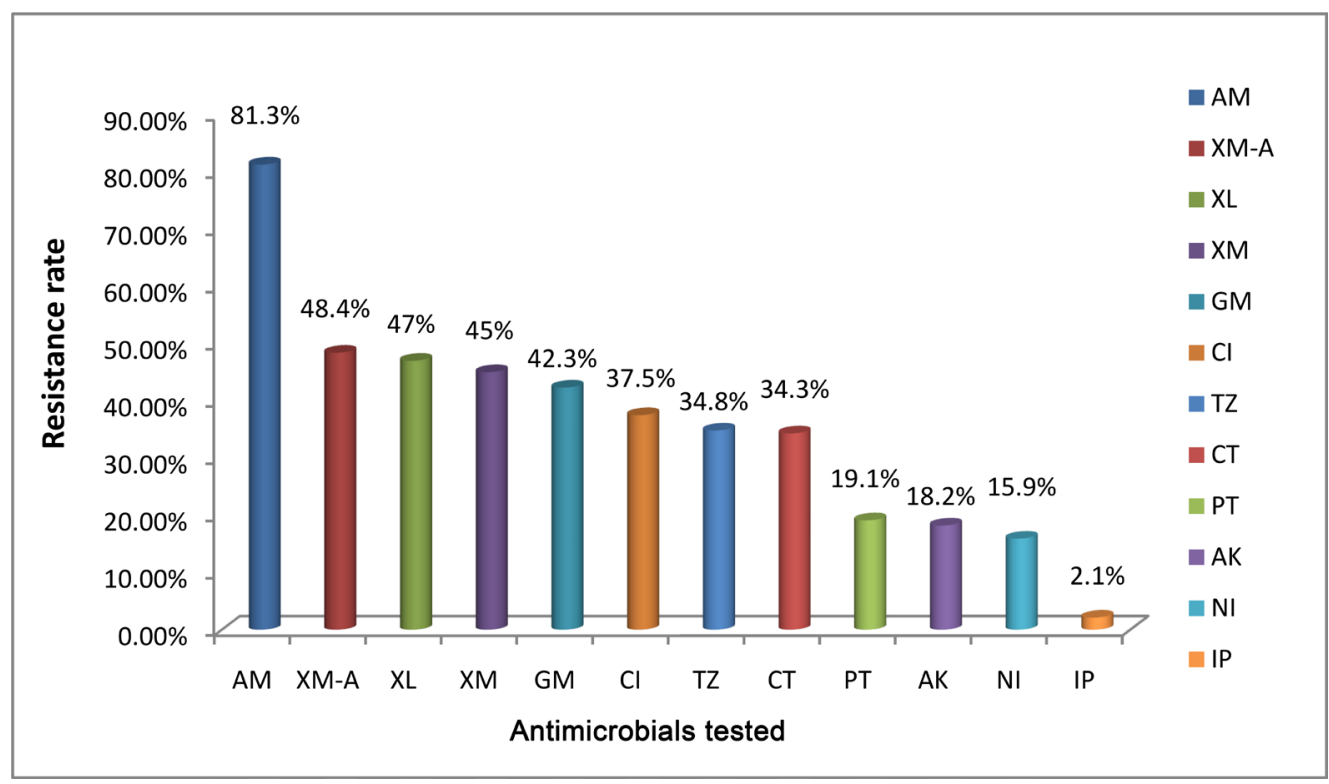

Key: $\mathrm{AM}=$ ampicillin; $\mathrm{XM}-\mathrm{A}=$ cefuroxime axetil; $\mathrm{XL}=$ amoxicillin/clavulanic acid; $\mathrm{XM}=$ cefuroxime; $\mathrm{GM}=$ gentamicin; $\mathrm{CI}$ = ciprofloxacin; $\mathrm{TZ}$ = ceftazidime; $\mathrm{CT}$ = cefotaxime; $\mathrm{PT}=$ piperacillin/tazobactam; $\mathrm{AK}=$ amikacin; $\mathrm{NI}=$ nitrofurantoin; IP = imipenem.

Figure 1. Resistance pattern of the 440 isolates to tested antimicrobials. 
The prevalence for ciprofloxacin resistance was 165/440 (37.5\%) using VITEK 2 and 161/440 (36.6\%) after determining the MIC using E-test.

\subsection{PCR Detection of PMQR Genes}

The PMQR genes were detected among 161 quinolone resistant Enterobacteriaceae distributed as shown on Table 2. There were 2/161 (1.2\%) strains with qnrA, 31/161 (19.3\%) strains with $q n r B, 13 / 161$ (8.1\%) strains with $q n r S, 84 / 161$ (52.2\%) strains with aac (6) Ib-cr, and 3/161 (1.9\%) strains with qepA.

The detection of the PMQR genes was compared among the important isolates, Klebsiella and Escherichia. The difference in prevalence was statistically significant in $q n r B$ and $q n r S$ with p-values $<0.0001$ as shown on Table 2.

\subsection{DNA Sequencing and Molecular Subtyping}

There were several mutations in the parC gene of Klebsiella leading to S80D and S80N substitutions. Two pairs of Klebsiella p pneumoniae strains were phenotypically and genotypically identical with $100 \%$ similarity in the dendrogramme. Furthermore, all E. coli strains were non diarrhoeagenic strains.

\section{Discussion}

This study showed that the resistance to ciprofloxacin was high with a resistance rate of $161 / 440(36.6 \%)$. This value is higher than that obtained among Gram-negative bacilli by Gangoué-Piéboji and collaborators who found 60/522 (11.5\%) among Gram-negative bacilli in Yaounde [10]. In Ghana, Namboodiri and collaborators also found a lower value, 40/293 (13.7\%), of quinolone resistance among E. coli [15]. In Greece, a lower value was also obtained among the Enterobacteriaceae with 266/1703 (15.6\%) of the isolates being quinolone resistant [16]. This high resistance rate could be due to the extensive use of fluoroquinolone for the treatment of pathologies such as urinary tract infections, respiratory tract infections, bone and skin infections, diarrhoea and even in the

Table 2. Detection and diversity of plasmid-mediated quinolone resistance gene among the 161 quinolone resistant Enterobacteriaceae.

\begin{tabular}{ccccc}
\hline & \multirow{2}{*}{ PMQR Gene } & \multicolumn{2}{c}{ Prevalent rate } & \multirow{2}{*}{ p-value } \\
\cline { 3 - 4 } & E. coli $(\mathrm{n}=68)$ & Klebsiella $(\mathrm{n}=56)$ & \\
\hline 1 & $q n r A$ & $0 \%$ & $2 / 56(3.6 \%)$ & $>0.3$ \\
2 & $q n r B$ & $4(6.25 \%)$ & $21(37.5 \%)$ & $<0.0001$ \\
3 & $q n r S$ & $3(4.4 \%)$ & $10(17.9 \%)$ & $<0.0001$ \\
4 & aac (6) Ib-cr & $33(48.5 \%)$ & $44(78.6 \%)$ & $<0.001$ \\
5 & $q e p A$ & $2(1.5 \%)$ & $1(1.8 \%)$ & $>0.9$ \\
6 & GyrA & $51(75 \%)$ & $51(91.1 \%)$ & $<0.002$ \\
7 & parC-E. coli & $68(100 \%)$ & $/$ & $/$ \\
8 & parC-Klebsiella & $/$ & $54(96.4 \%)$ & $/$
\end{tabular}


production of agricultural feeds like poultry feed.

The resistance of the isolates to ampicillin was the highest. Most of the isolates, $81.3 \%$ (358/440), were ampicillin resistant. This high resistance rate is similar to the 83\% obtained in Yaounde in 2004 [10]. The very high resistance could be attributed to the extensive use of this drug in the treatment of a variety of common infections; its availability without a medical prescription from non-registered pharmacies (road-side vendors); and its easy administration that is orally.

High resistance rates of above $30 \%$ were equally observed for the beta-lactamase inhibitor combination with amoxicillin/clavulanic acid having 214/440 (48.4\%) resistant isolates. The cephalosporins: cefuroxime 198/440 (45\%); cefotaxime 151/440 (34.3\%); and ceftazidime 153/440 (34.8\%) also showed high resistant rate of above $30 \%$. Piéboji and collaborators in 2004 found resistance rates below $20 \%$ among the cephalosporins. This shows that there has been an increase in resistance with time. Many factors may have contributed to this increase in resistance such as the extensive use of antibiotics, poor drug quality, and unhygienic conditions in the hospitals and environment leading to the rapid spread of infections. Lack of efficient surveillance in developing countries could also account for this lack of information and control mechanisms.

This study confirmed that the resistance among the carbapenems remained low especially with imipenem $9 / 440(2.1 \%)$ of the isolates being resistant. This may be because they have been reserved only for hospital use in multidrug-resistant (MDR) bacteria since they can only be administered intravenously.

There are various mechanisms responsible for resistance to fluoroquinolone (FQ) in the family of Enterobacteriaceae. In addition to the mutations observed in chromosomal genes which encode for DNA gyrase and topoisomerase IV, plasmid mediated quinolone resistance (PMQR) has equally been detected. The $q n r$-mediated inhibition of quinolone binding to DNA, the efflux pump encoded by $q e p A$ and also but not limited to the aac (6)-Ib-cr mediated quinolone acetylation [17]. We investigated the presence of $q n r A, q n r B, q n r S$ for qnr genes, the only aminoglycoside acetyltransferase variant ( $a a c(6)-I b-c r)$ and one of the efflux pump, qep $A$ using the conventional PCR method.

For the $q n r A$ gene, we found that of all isolates tested only two were positive and they were both Klebsiella isolates, 2/56 (3.7\%). Similar findings were obtained by Ajayi and colleagues in Nigeria in E. coli with only 1/20 (5\%) [18] while Fortini and colleague did not find any $q n r A$ in commensal E. coli from animal in Nigeria [19]. However, in Egypt 30\% of the E. coli isolates from hospitalized patients tested were positive for $q n r A$ in 2014 [20]. Outside Africa, Minh Vien and colleagues found 4/66 (6.1\%) among the Klebsiellae isolates from hospitalized patients and none among the community group [3]. In the United States of America, 8/72 (11.1\%) of Klebiellae pneumoniae isolates were positive for the $q n r A$ gene [21].

In this study, $q n r B$ was detected in Klebsiellae spp: 21/56 (37.5\%); Enterobac- 
ter spp: $4 / 7$ (57.1\%) Escherichia coli: 4/68 (5.9\%) and in the two Salmonella spp tested (100\%). The prevalence was higher in Egypt, with $61 \%$ of $E$. coli isolates among hospitalized patients being positive [20], whereas in Ghana, 2/40 (5\%) of $E$. coli haboured the $q n r B$ genes [15]. This was slightly lower than the values obtained in this study. Also 10/17 (58.8\%) quinolone resistant E. coli carried qnrB. The values were however higher from a hospitalized group of patients in Vietnam in 2009: Klebsiella spp 8/66 (12.1\%) and E. coli 1/55 (1.8\%) [3]. The qnrB was also detected in other species like Enterobacter and Citrobacter 2/28 (7.1\%) by Minh Vien and colleagues.

The qnrS was first detected in 2003 and it is related to the $q n r A 1$ by having $59 \%$ amino acid similarity. The presence of qnrS is able to confer up to 4 -fold increase in the MIC of nalidixic acid and about 30-fold increase in the fluoroquinolones [9]. The qnrS gene was identified in only 13 isolates: Klebsiella 10/56 (17.9\%) and $E$ coli 3/68 (4.4\%). This value was lower than that obtained in Ghana $7 / 40(17.7 \%)$ in quinolone resistant $E$. coli isolates [15]. A similar value was obtained in Egypt with 12/80 (15\%) of E. coli from hospitalized patient [20]. A significantly higher value was demonstrated in Vietnam among Klebsiella isolates from hospitalized patients 52/66 (78.8\%) [3]. However, lower values were obtained in Jamaica, 9.6\% [7]. Surveys carried out in Europe suggest that qnrS is more prevalent than qnrA [2]. This was confirmed in our study.

This study suggests that the PMQR genes particularly the qnr genes are widespread in our hospitals and communities. This dissemination may encourage the rapid development of fluoroquinolone resistance as they are capable of conferring low-level resistance to antimicrobials and promote the selection of high-level resistant strains with mutations on the chromosome including gyrA and parC [2]. This might lead to the primary source of selection for not just quinolone resistance but for the multidrug resistant bacteria. The use of antimicrobials in agriculture and in the production of animal feeds could also be another potential major source for selection of resistant organisms [3]. The aminoglycoside acetyltransferase variant, $a a c\left(6^{\prime}\right)-I b-c r$, can only confer very low levels of resistance which is most often lower than that confered by the qnr genes. The mechanism consists of altering the antimicrobial agent. Its main target is the piperzinyl secondary amine which is present in fluoroquinolones such as ciprofloxacin. Consequently, the quinolones which do not have the unsubstituted piperazinyl nitrogen are obviously not affected [9] [22]. When aac (6)-Ib-cr combines with $q n r A$ in the same cell the level of resistance increases by fourfold. Our study illustrates that the number of Enterobacteriaceae habouring the aac (6)-Ib-cr was higher than that habouring the qnr genes. We found out that 44/56 (78.5\%) of Klebsiella housed the aac (6) -Ib-cr gene. This prevalence was higher in Vietnam among Klebsiella species from hospitalized patients 11/66 (16.7\%) whereas only $1 / 45(2.2 \%)$ was detected from the community group (Minh Vien et al., 2009). The values obtained in the United States of America were also lower with 88/313 (28\%) of Enterobacteraceae isolates habouring the aac (6) -Ib-cr gene [23]. The 
prevalence was also lower in Sweden, 35/414 (8.4\%) [24].

In this study, 33/68 (48.5\%) of $E$. coli isolates housed the aac (6)-Ib-cr gene. This value was relatively higher than that obtained by Minh Vien and colleagues [3], 9/55 (16.4\%) and Tarchouna and colleagues, 12/36 (33\%) [25]. We were able to detect this gene in other Enterobacteriaceae like Enterobacter 4/7 (57.1\%), Pantoea 1/15 (6.7\%) Proteus and Salmonella. Minh and colleagues also detected it in one other Enterobacteriaceae species other than Klebsiella or E. coli [3]. This high prevalence and widespread of the $a a c(6)-I b$-cr gene probably reflects a long-time emergence and still ongoing dissemination of underdetected aac (6) $-I b-c r$ in the community [24].

Similarly, the efflux pump is also able to confer resistance in exporting quinolones out of the cell [26]. The efflux mechanism can act on single or multiple antimicrobial agents and is usually conferred through plasmids and chromosomes [9]. The prevalence of the qepA gene was very low in our study with just $2 / 68$ (2.9\%) and 1/56 (1.8\%) among quinolone resistant E. coli and Klebsiella pneumoniae isolates respectively. This low prevalence was also found in previous studies carried out in Africa and the rest of the world; Namboodiri and colleagues in Ghana had a similar report with $2 / 40$ (5\%) of quinolone resistant E. coli. Minh Vien and colleagues found that $1 / 55$ (1.8\%) of $E$. coli from a hospitalized group of patient housed the qepA gene and none among the Klebsiellae and none among the community group of patients which was made up of $340 \mathrm{E}$. coli and 45 Klebsiellae isolates [3]. In some studies no qep $A$ gene was detected at all. Guillard and colleagues found none out of 118 Enterobacteriaceae isolates using the real-time PCR method [4].

Very little information is available about the epidemiology of the newly discovered PMQR pump qepA gene. A survey carried out in Japan found $q e p A$ in 2 $(0.3 \%)$ of 751 E. coli isolates collected from 140 hospitals between 2002 and 2006 [27]. The qepA gene has also been detected in isolates from chickens as demonstrated in Nigeria from 162 ampicillin-resistant $E$ coli with three isolates housing this gene [19].

We carried out Pulsed-Field Gel electrophoresis (PFGE) on E. coli and Klebsiella pneumoniae pneumoniae isolates which were the most prevalent Enterobacteriaceae species to determine the relationship between strains through their genotypic characterization.

The PFGE pattern for the Klebsiella pneumoniae pneumonae strains showed that two pairs of strains (C054 and C057) were closely related with a $100 \%$ similarity. They were both isolated from the same hospital and the urine of babies in the same neonatal ward. Their antimicrobial resistance profile was also similar and equally housed the same PMQR genes. This close similarity could possibly mean they were the same strains which could have been transferred from one baby to the other since Klebsiellae species are primarily hospital pathogens causing nosocomial infection. Another, pair C162 and C361 of K. p. pneumoniae strains also showed a $100 \%$ similarity phenotypically and genotypically. This 
transfer of isolates from one patient to the other may be due to poor hygienic or aseptic conditions in the hospitals.

This study reports the first identification of PMQR among Enterobacteriaceae in Cameroon. It shows that the quinolone resistance is high and the plasmid-mediated quinolone resistant genes accounting for this resistance are diversified and include $q n r A, q n r B$, $q n r S$, aac (6) $I b-c r$, the efflux pump $q e p A$, with mutations occurring in the quinolone resistant determining region of $g y r A$ and $\operatorname{par} C$.

To avoid the rapid dissemination of these PMQR genes among Enterobacteriaceae isolates, the use of antimicrobials should be strictly controlled in both human and veterinary medicine. Continuous surveillance of antimicrobial resistance should be carried out in not only humans but also in animals to monitor these genes.

The limitation of the study lies in the selection of only three reference hospitals in Yaoundé. This does not allow us to generalize our results. Due to cost we were unable to carryout MIC for more than one FQ from a family of over 30 antimicrobials. We were unable to sequence all the quinolone resistant strains which haboured the qnr genes and to genotypically compare all quinolone resistant E. coli and Klebsiella pneumoniae strains.

\section{Conclusion}

This study shows that the quinolone resistance is high and the PMQR genes accounting for this resistance are diversified. This high PMQR indicates that there has been an unknown circulation of these genes in our community. In order to avoid the rapid spreading of these PMQR genes continuous surveillance of antimicrobial resistance should be carried out in not only humans but also in animals to monitor the evolution of these genes.

\section{Acknowledgements}

This study received funding from the Organization for Women in Science for Developing World (OWSD) and Swedish International Development Coorperation Agency (SIDA) PhD sandwich fellowship programme. The fund provided by OWSD was used to carry out the analysis at the Center for Enteric Diseases, National Institute for Communicable Diseases, Johannesburg, South Africa.

\section{Authors' Contributions}

EEL-M, TM, SKS conceived and designed the project. MT, M-COA, HKG, ACB, MTM, ABE, GMI carried out the phenotypic analysis. EEL-M, KHK, AMS, NC carried out the molecular analysis. EEL-M and MTM drafted the manuscript. All authors proof read and approved the manuscript.

\section{Conflicts of Interest}

The authors declare that they have no competing interests. 


\section{References}

[1] Lennete, H.E., Balows, A., Hausler Jr., W.J. and Shadomy, H.J. (1985) Manual of Clinical Microbiology. Fourth Edition, American Association of Microbiology Press, Washington DC, 263-277.

[2] Robicsek, A., Jacoby, G.A. and Hooper, D. (2006) The Worldwide Emergence of Plasmid-Mediated Quinolone Resistance. Lancet Infectious Disease, 6, 629-640. https://doi.org/10.1016/S1473-3099(06)70599-0

[3] Minh Vien, L.T., Baker, S., Thao, L.T.P., Tu, L.T.P., Thuy, C.T., Nga, T.T.T., Hoang, N.V.M., Campbell, J.L., Yen, L.M., Hieu, N.T., Chau, N.V., Farrar, J. and Schultsz, C. (2009) High Prevalence of Plasmid-Mediated Quinolone Resistance Determinants in Commensal Members of the Enterobacteriaceae in Ho Chi Minh City, Vietnam. Journal of Medical Microbiology, 58, 1585-1592.

https://doi.org/10.1099/jmm.0.010033-0

[4] Guillard, T., Moret, H., Brasme, L., Carlier, A., Vernet-Garnier, V., Cambau, E. and De Champs, C. (2011) Rapid Detection of qnr and qepA Plasmid-Mediated Quinolone Resistance Genes Using Real-Time PCR. Diagnostic Microbiology and Infectious Diseases, 70, 253-259. https://doi.org/10.1016/j.diagmicrobio.2011.01.004

[5] Livermore, D.M., James, D., Reacher, M., Graham, C., Nichols, T., Stephens, P., Johnson, A.P. and George, R.C. (2002) Trends in Fluoroquinolones Resistance in Enterobacteriaceae from Bacteremias, England and Wales, 1990-1999 Ciprofloxacin Research. Emerging Infectious Diseases, 8, 473-478.

[6] Pitout, J.D. and Laupland, K.B. (2008) Extended-Spectrum Beta-Lactamase Producing Enterobacteriaceae: An Emerging Public Health Concern. The Lancet Infectious Diseases, 8, 159-166. https://doi.org/10.1016/S1473-3099(08)70041-0

[7] Stephenson, S., Brown, P.D., Holiness, A. and Wilks, M. (2010) The Emergence of Qnr-Mediated Quinolone Resistance among Enterobacteriaceae in Jamaica. West Indian Medical Journal, 53, 241-244.

[8] Jacoby, G.A., Walsh, K.E., Milles, D.M., Walker, V.J., Oh, H., Robicsek, A. and Cooper, D.C. (2006) QnrB, Another Plasmid-Mediated Gene for Quinolone Resistance. Antimicrobial Agents and Chemotherapy, 50, 1178-1182. https://doi.org/10.1128/AAC.50.4.1178-1182.2006

[9] Strahilevitz, J., Jacoby, G.A., Hooper, D.C. and Robicsek, A. (2009) Plasmid-Mediated Quinolone Resistance: A Multifaceted Threat. Clinical Microbiology Review, 22, 664-689. https://doi.org/10.1128/CMR.00016-09

[10] Gangoue-Pieboji, J., Koulla-Shiro, S., Ngassam, P., Adiogo, D. and Ndumbe, P. (2006) Antimicrobial Activity against Gram Negative Bacilli from Yaounde Central Hospital, Cameroon. African Health Science, 6, 232-235.

[11] Toukam, M., Lyonga, E.E., Assoumou, M.-C.O., Fokunang, C.N., Atashili, J., Kechia, A.F., Gonsu, H.K., Mesembe, M., Eyoh, A., Ikomey, G., Akongnwi, E. and Ndumbe, P. (2010) Quinolone and Fluoroquinolone Resistance in Enterobacteriaceae Isolated from Hospitalised and Community Patients in Cameroon. Journal of Medicine and Medical Sciences, 1, 490-494.

[12] Lyonga, E.E., Toukam, M., Atashili, J., Gonsu, H.K., Adiogo, D., Mesembe, M., Nguefack-Tsague, G., Eyoh, A., Ikomey, G., Mukwele, B., Meli, T.J.M. and Okomo-Assoumou, M.-C. (2013) A Comparative Study on Susceptibility of Enterobacteriaceae to Six Quinolones in Yaounde. Health Science and Disease, 14, 1-7.

[13] Lyonga, E.E., Toukam, M., Nkenfou, C., Gonsu, H.K., Okomo-Assoumou, M.-C., Mesembe, M.T., Eyoh, A.B., Ikomey, G.M., Ndze, V.N. and Koulla-Shiro, S. (2015) Resistance Pattern of Enterobacteriaceae Isolates from Urinary Tract Infections to 
Selected Quinolones in Yaounde. The Pan African Medical Journal, 21, 105. https://doi.org/10.11604/pamj.2015.21.105.5469

[14] Clinical Laboratory Standard Institute (CLSI) (2016) Performance Standards for Antimicrobial Susceptibility Testing. Sixteenth Informational Supplement. Wayne.

[15] Namboodiri, S.S., Opintan, A.J., Lijek, S.R., Newman, J.M. and Okeke, N.I. (2011) Quinolone Resistance from Escherichia coli from Accra, Ghana. BioMed Central Microbiology, 11, 44. https://doi.org/10.1186/1471-2180-11-44

[16] Skandami-Epitropaki, V., Xanthaki, A., Tsiringa, A., Fotiou, P., Kontou, C. and Toutoua, M. (2008) Fluoroquinolones Resistance in Enterobacteriaceae Strains Isolated from Community Acquired Urinary Tract Infections. European Society of Clinical Microbiology and Infectious Diseases, 8.

[17] Tran, J.H., Jacoby, G.A. and Hooper, D.C. (2005) Interaction of the Plasmid-Encoded Quinolone Resistance Protein qnrA with Escherichia coli Topoisomerase IV. Antimicrobial Agents and Chemotherapy, 49, 3050-3052. https://doi.org/10.1128/AAC.49.7.3050-3052.2005

[18] Ajayi, A.O., Oluduru, A.O., Olowe, O.A., Odeyemi, A.T. and Famurewa, O. (2012) Plasmid Mediated Fluoroquinolone-Resistance $q n r A$ and $q n r B$ Genes among Escherichia coli from Cattles in Ado-Ekiti, Nigeria. West Indian Medical Journal, 61, 784-788.

[19] Fortini, D., Fashae, K., Gracia-Fernandez, A., Villa, L. and Carattoli, A. (2011) Plasmid-Mediated Quinolone Resistance and $\beta$-Lactamases in Escherichia coli from Healthy Animals from Nigeria. Antimicrobial Chemotherapy, 66, 1269-1272. https://doi.org/10.1093/jac/dkr085

[20] El-Sokkary, M.M.A. and Abdelmegeed, E.S. (2014) Antimicrobial Resistance Pattern among Escherichia coli Strains Isolated from Mansoura Hospitals in Egypt with a Special Reference to Quinolones. African Journal of Microbiology Research, 9, 662-670. https://doi.org/10.5897/AJMR2014.7351

[21] Wang, M., Sahm, D.F., Jacoby, G.A., Wang, F. and Hooper, D.C. (2004) Emerging Plasmid-Mediated Quinolone Resistance Associated with qnr Gene in Klebsiella pneumoniae Clinical Isolates in the United States. Antimicrobial Agents Chemotherapy, 48, 1295-1299. https://doi.org/10.1128/AAC.48.4.1295-1299.2004

[22] Guan, X., Xue, X., Liu, Y., Wang, J., Wang, Y., Wang, J., Wang, K., Jiang, H., Zhang, L., Yang, B., Wang, N. and Lei, P. (2013) Plasmid-Mediated Quinolone Resistance-Current Knowledge and Future Perspectives. Journal of International Medical Research, 41, 20-30. https://doi.org/10.1177/0300060513475965

[23] Park, C.H., Robicsek, A., Jacoby, G.A., Sahm, D. and Hooper, D.C. (2006) Prevalence of aac(6')-Ib-cr Encoding a Ciprofloxacin-Modifying Enzyme. Antimicrobial Agent and Chemotherapy, 50, 3953-3955. https://doi.org/10.1128/AAC.00915-06

[24] Karah, N., Poirel, L., Bengtsson, S., Sundqvist, M., Kahlmeter, G., Nordmann, P., Sundsfjord, A., Samuelsen, O. and The Norwegian Study Group on PMQR (2010) Plasmid-Mediated Quinolone Resistance Determinants $q n r$ and aac(6)-Ib-cr in Escherichia coli and Klebsiella spp. from Norway and Sweden. Diagnostic Microbiology and Infectious Diseases, 66, 425-431. https://doi.org/10.1016/j.diagmicrobio.2009.12.004

[25] Tarchouna, M., Ferjani, A., Marzouk, M., Guedda, I. and Boukadida (2015) Prevalence of Plasmid-Mediated Quinolone Resistance Determinants among Clinical Isolates of Escherichia coli in a Tunisian Hospital. International Journal of Current Microbiology and Applied Science, 4, 195-206.

[26] Zurfluh, K., Abgottspon, H., Hächler, H., Nüesch-Inderbinen, M. and Stephan, R. 
(2014) Quinolone Resistance Mechanism among Extended-Spectrum Beta-Lactamases (ESBL) Producing Escherichia coli Isolated from Rivers and Lakes in Switzerland. PLOS ONE, 9, e95864. https://doi.org/10.1371/journal.pone.0095864

[27] Yamane, K., Wachino, J., Suzuki, S., Kimura, K., Shibata, N., Kato, H., Shibayama, K., Konda, T. and Arakawa, Y. (2007) New Plasmid-Mediated Fluoroquinolone Efflux Pump, qepA Found in an Escherichia coli Clinical Isolate. Antimicrobial Agent and Chemotherapy, 51, 3354-3360. https://doi.org/10.1128/AAC.00339-07 